\title{
Index of Names and Terms
}

\author{
Abdallah ibn Salam $\quad 37,39-47$ \\ Abraham 179, 181, 197, 207, 209-210 \\ Abū Ḥanīfa 223-224 \\ Abū Hurayra 191, 219 \\ Abū Rā’ița al-Takrīīī $\quad 216$ \\ Alan of Lille $\quad 6,105,107$ nn. 2-3, 108, 116 \\ Al-'Aqqad, 'Abbas 199-200 \\ Al-Dajjal 191-192 \\ Al-Fārābī $\quad 227$ \\ Al-Ghazali, Abu Hamid 194 \\ Al-Ḥallāj 233 \\ Al-Husayn $\quad$ 197-198 \\ 'Ali $\quad 197-198$ \\ al-ihsan 186 \\ Al 'Imran 40, 184 \\ Al-Masih, 'Isa 179 \\ Altaner, Berthold $\quad 168$ n. 30 \\ Al-Wahidi 44 \\ Amadis de Gaule 174 \\ Arabic language $\quad 162$ \\ Armenians 108 \\ Augustine $59,70-71,73$ ก. 34, 81-83, 96, 119, \\ 121,156
}

Baghdad 106, 113, 119, 125, 136

Baldini, Ugo 173 n. 53

baptism 58, 94, 101, 105, 109-110, 112, 116, 120

Bartoli, Daniello $\quad$ 169, 172

Bartolomeo Piceno de Montearduo 130

basmala 116-117

bātin (hidden) 220-221

Bellitto, Christopher M. 49 n. 3

Bessarion, Basilios 123 n. 1, 142

Bibliander (Theodor Buchmann) 37

Biechler, James E. $\quad 1,21,37-38,56$ n. 34, 64 n. 72,69 n. 5,82

Bisaha, Nancy $\quad 123,129$ n. 28, 139

blasphemy $\quad 162,164,170-171,241,251$

Blum, Paul Richard $\quad 6,89$ n. 12,

Boethius 76, 99

Bonner, Anthony $160 \mathrm{n} .2$

Bovelles, Charles de $\quad 165$

Boyle, Marjorie O’Rourke 168 n. 31
Broderick, James 175 n. 63

Burman, Thomas E. 5,46 n. 45,121 n. 64,123 , $125,143,144$ n. 85,147

Calderón de la Barca, Pedro 170

Câmara, Louis Gonçalves da 168 n. 32

Candellero, Massimo 162 n. 8

Carrèras y Artau, Tomás and Joaquin 163 n. 11

Cathars 107-108, 110

Cervantes, Miguel de 174

Christianson, Gerald $11 \mathrm{n} .14$

Christology $\quad 5^{-6,53}, 54$ n. 20, 55-56, 59, 69, $75^{-76}, 78-80,84-85,102,162,164$

circumcision $\quad 58,105,108-109,112-113$, $118-120$

Classen, Joachim Carl 132 n. 40

Constantinople $2,10,17,20-22,30-31,34$, $39,49-51,68,70,74,88$ n. $6,111,113$ n. 32, 132-135, 145

Corsi, Giuseppe 127 n. 17

Cribratio Alkorani $\quad$ 1, 6, 10, 12-15, 18, 20-21, 23, 27-29, 33-39, 45, 47, 51, 61, 71-72, $76-77,95,111,124,135^{-136,138-139}$

Daiber, Hans $\quad 164$ n. 16

Daniel, Norman 34 n. 13

De docta ignorantia $6,18,24,51,53-55$, 59-6o, 63, 73, 97

De pace fidei $1-3,6,10,12-13,15,18,20-27$, 29-35, 37-39, 48, 51, 54, 57 n. 41, 58-63, $68-72,73$ n. $34,74-85,88,95,97-98,107$, $111,113,137-138$

devshirme $\quad 151$

Domínguez Reboiras, Fernando 162 n. 6 Dougherty, Michael 166

Eckhart $\quad 235^{-251}$

Ecumenical Councils 76

Erasmus of Rotterdam 170

Eschatology 204, 208

Ethiopian Orthodox 113

Euler, Walter Andreas 86 n. 1, 88 n. 7, 113 n. 31,163 n. 12 
Ficino, Marsilio $\quad$ 124, 135, 139

Francis of Assisi 175 n. 66

Friends of God 196-197

Gabriel (Angel) 42-43, 45, 180, 182, 186, 209, 237, 239-241, 243

Gaeta, Franco 132 n. 40

Gautier, Léon 174 n. 57

Gayà, Jordi 161 n. 4

George of Trebizond 124, 129, 132-134, 142

Gilbert Crispin $\quad$ 108, 111, 121-122

Ḥasan al-Basrī 232

Hadith $42-43,47$ n. 48, 117 n. 48, 191-192, 200, 204-205, 218-219, 223, 246

Hagemann, Ludwig $\quad 12$ n. 20, 14, 16-18, 38-39

Hanafi 116, 223

Hankins, James 124 n. 4

Hermann of Dalmatia $\quad 36,38-40,45-46$

Hick, John 31

Hunt, Lynn 173 n. 53

Ibn 'Abbas $\quad 185$

Ibn 'Arabi $\quad$ 5-6, 196-197, 236-242, 244-247, 249-251

Ibn Ḥazm 4, 220, 222-227, 229-230, 233

Ibn Hisham 40, 194

Ibn Ishaq 40

Ibn Kathir $\quad 43-44$

Ibn Masarra 221

Ibn Sīnā 227

Ibn Taymiyya 221 n. 8, 225, 227, 230

Ignatius of Loyola $\quad 5^{-6,160-161,168}$

Incarnation $29,52-54,59-60,72,74,80,98$, 108, 155, 158, 164, 186-187, 193, 205, 209, 212, 233, 241, 249-251

Isa ibn Maryam (Jesus son of Mary) 4 n. 13, 6, 62 n. 63, 89-91, 157, 179-201, 219, 230, 241-242

Islam $1-4,6,9-10,12-14,16-24,26-27$, 29-31, 34-37, 39-40, 42-44, 46-47, 51, $5^{6}$ n. 33, 6o, 63, 67-69, 71, 76-80, 83-85, 88 nn. 6, 9, 89, 92, 94-95, 102, 105-106, 109, $111,113,114$ n. 37, 116 n. 45, 117, 120-132, 134-135, 136 n. 56, 137-138, 141-143, 145-152, $155,179,180$ n. 2, 181 nn. 3-4, 183-184, 189-190, 193-194, 197, 199-205, 207-208, 211, 213, 215-216, 219, 221, 223, 225, 229 n. 32, 233-234, 236, 238, 246, 250 n. 32, 251
Izbicki, Thomas M. 88 n. 9, 153

Izzi, Pierangela 128 n. 19

Jacob, Margaret C. 173 n. 53

Jacobites 107, 113

Janissaries $\quad 151$

Jean Germain $\quad 145$ n. 1, 146, 147 n. 9, 150

Jews, Judaism $\quad$ 105-113, 119-122

John of Damascus 20 n. 1, 216

Johnston, Mark D. 161 n. 5, 168 n. 30

Juan de Segovia $\quad 6,9,145,147$ n. 9, 150-151

Judah Halevi 46

Judaizing 110, 113, 120

Ka'ba 194

Karbala $\quad 197-198$

Khalid, Muhammad Khalid 200

Khalidi, Tarif $\quad$ 193, 201, 240 n. 11

Kianka, Frances 129 n. 29

knighthood $\quad 172-173,175$

Kristeller, Oskar Paul 139 n. 71, 140 n. 73

Kydones, Demetrius $\quad$ 124, 129, 130 n. 32, 131 n. 38,134

lafza, pl. alfäz (word) 223-224

learned ignorance $33,75,79$

Leturia, Pedro 169 n. 34, 170 n. 41

Llinàs Puente, Carles 163 n. 11

Logos $69,71,76,80,85,88$ n. 8,186 n. 18,187 , 197

Lull, Raymond $\quad 160$ n. 1, 161-168, 171-172, 174 n. 57

Mallette, Karla $\quad 127$ n. 18

ma'nā, pl. ma'ānī (meaning) 213, 218

manuductio $\quad 23,95,98$ n. 55

Mary 6, 24, 28 n. 28, 62, 89-91, 157, 161, 170, 174 n. 57, 175, 180-193, 206, 219, 230-231, 237, 239, 241-243

Maryam (see Mary)

masa'il (questions) 39

Mazzucchi, Carlo Maria 134 n. 53

Mecca 27 n. 27, 194, 223

Mercati, Giovanni 129 n. 29, 130 n. 30

Mérigoux, Jean-Marie 114 n. 33, 125 n. 7, 130 n. 30,134 n. 51,136

Meserve, Margaret 123 n. 3

Meuthen, Erich 10 n. 7,38 n. 23, 39, 50 n. 5 , 145 n. 2,149 n. 23 
Mijnhardt, Wijnand $\quad 173$ n. 53

mikveh (Jewish ritual washing) 105, 120

Monfasani, John 132 n. 40, 133, 135

monotheism $45,51^{-} 5^{2}, 55,57$ n. 41, 6o-61,

94, 97, 100, 179, 207, 238

Montserrat $\quad 161,168-169,171,173-174$

Moors $\quad 162$ n. 6, 169

Moriscos $\quad 169$ n. 36

Mosul 114, 118

Mother of the Book (Umm al-Kitāb) 209-210

Muhammad (Prophet) 37, 39-40, 43 n. 35, 180, 191, 194, 198, 200, 219

Muslims (see also Saracens) 2, 4, 6, 15, $17-19,23-25,28,35-36,41-42,44,46,49$, 56 n. 32, 57, 59 n. 51, 61-63, 68-69, 72, $83-85,87$ nn. 2-3, 88-89, 94-95, 98-99, 105, 107-108, 110-112, 114-121, 124-126, 130-131, 134-136, 138-161, 164 n. 17, 167-168, 170, 173, 175, 179-180, 190-191, 194, 197, 200-202, 205-206, 209, 213 n. 19, 218-219, 225, 231, 233, 235, 236 n. 3, 236 n. 3, 238, 242

Nederman, Cary J. 123

Nestorians 107

Nicholas of Cusa 2, 4 n. 12, 12, 20-27, 29-34, $36-40,45,47,49$ n. 1,65 n. $75,68,69$ n. 6 , 75 n. 49, 85-86, 88, 98, 100, 105, 111-113, 121 n. $63,136-139,145$

niyya (intention) 220, 223, 229

Obermeier, Anita $\quad 161 \mathrm{n} .4$

Olesh, Andrew 175 n. 66

Olshki, Leonardo 127 n. 18

Peers, E. Allison $\quad 165$ n. 19

Pertusi, Agostino $\quad 134$ n. 53

Peter the Venerable $10,36-37,39,107,109$

Petrus Alfonsi 114 n. 36-37, 116, 121

pia interpretatio $14-15,27,95,137$

Piccolomini, Silvio Enea 14 n. 29, 123 n. 1, 135 n. 54,146

Piemontese, Michele Angelo $\quad 123$ n. 3, 139

Platzeck, Erhard Wolfram $\quad 161,165$

Plethon, Gemisto George 129, 142

Pliny the Elder 128

Polonio, Martin 128

Pontanus, Jacobus $\quad 170,171$ n. 44
Preserved Tablet, see Mother of the Book (Umm al-Kitāb)

Proclus 97

Pseudo-Methodius 133, 135

Quesnel, Pierre $\quad$ 173-174

Qur'an 1-2, 4-6, 9-10, 13-16, 18, 20-21, 24-31, 33-39, 41, 43-45, 56 n. 32, 57, 59 n. $51,61-63,67,72,74,83,86,89-90,95$, $109,111,115$ n. 40,116 n. 45, 117-118, 121-122, 124-129, 134-135, 137-141, 143-148, 150 n. $28,154-155,158,179-184,186-189,191$, 199, 203-219, 222-223, 229, 231, 235-237, 243

Rābi‘a al-'Adawiyya $\quad 232$

Ramon Martí 114 n. 37, 119, 121

Ravegnani, Giorgio 132 n. 40

Raymond of Sabunde 99

Reiss, Edmund 142

religio una in rituum varietate $30 \mathrm{n} .1$, 32 n. $6,38,40,49,60,64$ n. 72, 71-73,

111 n. 24

revelation (of Qur'ān) $\quad 216$

Ribadeneira, Pedro 169

Riccoldo da Montecroce 4, 6, 37 n. 20, 106, 108, 113-122, 124-129, 136 n. 56

rite $\quad 58-59,81,112,153$

Rizzardi, Giuseppe 125 n. 9

Robert of Ketton 9, 14, 20-21, 37-38, 109

Ryder, Judith R. 130 n. 29-30, 131 n. 33-35

Ševcenko, Ihor 130 n. 29

shirk 90-91, 95, 99

Sira 204-205

slaves 150

Smith, Wilfred Cantwell $\quad 31,82$

Suárez, Francisco 170

Sufism (Islamic mysticism) $\quad 232$

Supersessionism 180 n. 2, 217

Tabdīl 214-215, 221 n. 8

Tahriff(corruption of scripture) $\quad 213$

Talmud 119

Tartars (Mongols) 10, 107, 108 n. 6

theology of religion $\quad 22-29,30-31,47-48$, 54-6o, 63-67, 84, 98, 122

Thierry of Chartres $\quad 97$

Thomas Aquinas $12,96,130,170$ 
Tolan, John Victor 3 n. 9, 127 n. 18, 147 n. 13 , 151 n. 36,175 n. 66

Toledan Collection $\quad 31,37-39,45,109$

translation (of Qur'an) 2, 5, 9-10, 14-15, 20, $24-25,37-38,43,109$

Trinity $13,23-24,29,52-55,57,59-62,66$, $72,86,88-92,94-99,102,108,110,126$,

134-135, 155, 158, 205-206, 212, 216, 219, 231

uns (intimacy) $\quad 232$

Valkenberg, Pim 36 n. 16,46 n. 42, 136 n. $5^{6}$, 137 n. 66,138 n. 67,235 n. 1

Vasoli, Cesare 139 n. 71

Vega, Amador 162 n. 8
Villani, Filippo 127 n. 18

violence $32,49,63,67,70,74,84,87,111,126$, $128,131,136,139^{-140}, 165^{-167}, 175^{-176}$

virginity 161,170

Volf, Miroslav 30,68 n. 1

Waldensians 107-108

Weiss, Roberto 134 n. 53

Wisdom Word 69, 83

wudu (Muslim ablutions before prayer) 109-110

zāhir (apparent) 220

Zāhirī school 220

zeal $32,165,172$ 